\title{
Crystal Growth of the Acentric Organic Nonlinear Optical Material Methyl-p-hydroxybenzoate: Morphological Variations in Crystals Grown by Physical Vapor Transport
}

Wenbo Hou, ${ }^{1}$ Radoljub I. Ristic, ${ }^{2}$ Karuppannan Srinivasan, ${ }^{3}$ Ranko M. Vrcelj, ${ }^{4}{ }^{\circ}$ Robert B. Hammond, ${ }^{5}$ David B. Sheen, and John N. Sherwood*(1)

WESTCHEM, Department of Pure and Applied Chemistry, University of Strathclyde, Glasgow G1 1XL, Scotland, U.K.

Supporting Information

\begin{abstract}
Single crystals of the acentric compound methyl-p-hydroxybenzoate were grown by self-nucleation and seeded growth from the vapor phase by the physical vapor transport (PVT) process. In the temperature range of $80-95{ }^{\circ} \mathrm{C}$ (nucleation supersaturation 0.97 to 0.88 ), all crystals were of the polymorphic form as produced by roomtemperature solution growth. Self-nucleated crystals varied in macromorphology from columnar to octahedral to skewed octahedral and finally to skewed columnar but retained the same crystal forms indicated by theoretical calculations.

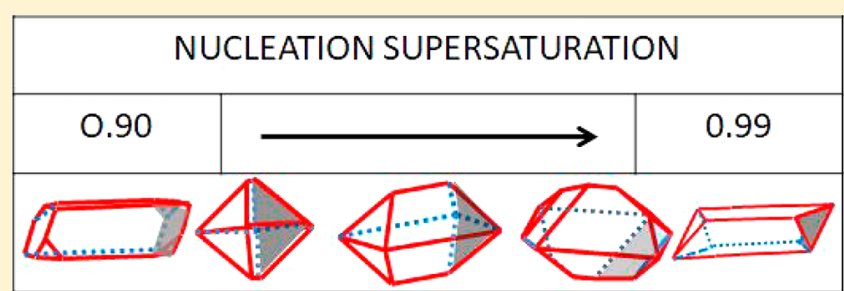

Shaded end - No growth [001] Micromorphological studies of growth faces indicated that these variations result from changes in growth mechanisms that influence both the defect structure and perfection of the growing crystal. X-ray topographic studies confirmed that growth under the most ideal conditions, when the dominant faces of the crystals were growing by a dislocation induced Burton, Cabrera, and Frank mechanism, yielded the structurally most perfect crystals. Preliminary studies of seeded growth were performed as a prelude to using PVT for the growth of larger crystals. The seeded growth followed a different pattern of supersaturation dependence. All crystals showed the same asymmetric growth along the polar axis that has come to be regarded as characteristic of these highly polar acentric materials when grown from solution.
\end{abstract}

\section{INTRODUCTION}

The requirement for highly efficient nonlinear optical (NLO) materials for use in the fabrication of optical switching and amplification devices in the areas of optical processing and communication has led to the investigation of the potential of organic materials for this purpose. ${ }^{1-3}$ The essential structural requirement for the materials to be useful is that the molecules should crystallize into an acentric crystallographic form: also that the material should be both structurally and chemically stable in the region of temperature over which the optical devices would be expected to operate. For their full optical evaluation, the crystals should also be capable of being prepared in as high an optically and structurally perfect form as is possible. Much effort has been expended on the formulation of the required molecular and structural basis for high nonlinear optical performance. ${ }^{4-6}$ This has resulted in the realization that the basic requirements are fulfilled by highly polar organic molecules when assembled into the acentric structure, the highest efficiencies of operation being achieved when the constituent molecules are mutually aligned in very specific orientations. ${ }^{4}$ These discoveries have directed the design and synthesis of a large range of novel organic optoelectronic materials. The success of this effort and its predictive capacity represents one of the major achievements in the field of crystal engineering.
The full assessment of the optical properties and subsequent production of an operable device still requires the growth of large optically transparent crystals. Methods of achieving such crystals by the engineering of the solution growth process using seed selection techniques ${ }^{7-11}$ have helped to overcome this problem. There still remains however a major problem in the growth of this type of crystal that needs to be understood.

The problem is that some highly polar, acentric, organic materials show a unique property; they grow only in one polar direction and then not always perfectly. This behavior was recorded first by Wells ${ }^{12-14}$ in the 1940s following experiments on the variation in morphology of acentric $\alpha$-resorcinol crystals when grown from different solvents. Wells concluded that the differential growth arose from the different degrees of solvent adsorption on the negative and positive faces of the crystal thus inhibiting growth to differing extents. This quite reasonable proposal has held sway for the ensuing 70 years due to a wealth of support gathered from both theoretical and experimental studies. ${ }^{15-21}$ It led to the general belief that the habits of crystals could be controlled by solvent selection on the basis of solventsurface interactions. In 2006, while searching for methods of

Received: November 19, 2018

Revised: $\quad$ August 28, 2019

Published: September 9, 2019 
overcoming the disadvantageous unidirectional growth problem, it was argued that if solvent inhibition was the root cause of the problem, then growth from the vapor phase, with no solvent present, should yield equivalent growth from both polar ends of the growing crystal. Surprisingly, crystals of $\alpha$-resorcinol grown from the vapor phase showed the same unidirectional growth behavior as those grown from solution. ${ }^{22}$ Later experiments ${ }^{23}$ confirmed that the differential in growth rates between the positive and negative faces was mechanistically based. This discovery has been followed by several theoretical studies of the growth of $\alpha$-resorcinol to account for this behavior. ${ }^{24-27}$ These relied on the particular crystallization behavior of this material and did not extend to the generality of this behavior among highly polar acentric materials. It remains one of the outstanding unsolved problems of crystal growth to define more broadly why this difference should arise.

To achieve this understanding requires the detailed examination of the physical vapor transport (PVT) growth of a wider range and types of acentric materials than have been studied. $^{22,23}$

To this end and to spread types of materials examined to the highly polar NLO materials we initiated a comparison of the growth behavior of the NLO material methyl-p-hydroxybenzoate (MHB) from both solution and the vapor phase. Additional aims were to define the growth behavior as a prelude to the development of a vapor growth method for the production of large single crystals; also to compare the results of the vapor and solution growth processes, which to some extent have been regarded as mechanistically different. Success in these aims could also lead to opportunities to prepare satisfactory crystals of a wider range of materials the general properties of which preclude solution growth such as, for example, polymorphs, the stability regions of which lie outside the normal temperature ranges accessible to growth from solutions.

The title material (MHB, Figure 1a) has been identified as a promising NLO material. The high second harmonic generation efficiency of powder samples was first reported by Jerephagnon in $1970 .^{28}$ Much later Sreeraman et al. ${ }^{29}$ measured this to be as much as 40 times that shown by urea, and $\mathrm{Li}$ et al. ${ }^{30}$ measured the optical coefficients $d_{\mathrm{ij}}$ and $d_{\text {eff }}$ of single crystals grown from solution. These results and a further optical examination by Lakshmanna Perumal et al. ${ }^{31}$ have confirmed the optoelectronic potential of this material. Previous studies of crystal growth of MHB have been restricted to sample preparation, principally for the purposes of the above optical studies, using standard solution growth techniques. More recently it has been shown that melt growth using the Czochralski technique can also be successful. $^{32}$

No detailed studies have been made of the growth process, and no previous study has indicated other than normal isotropic growth behavior for this material. The material has a higher than usual vapor pressure, a property that could help in the control of the vapor phase growth process and makes it an ideal candidate for PVT growth.

It is also interesting to note that MHB (under the commercial name Nipagin $\left.{ }^{(}\right)$finds widespread use as an antifungal and preservative agent in cosmetics, foods, and drugs. ${ }^{33}$ Workers involved in these areas may also find interest in the results of these studies.

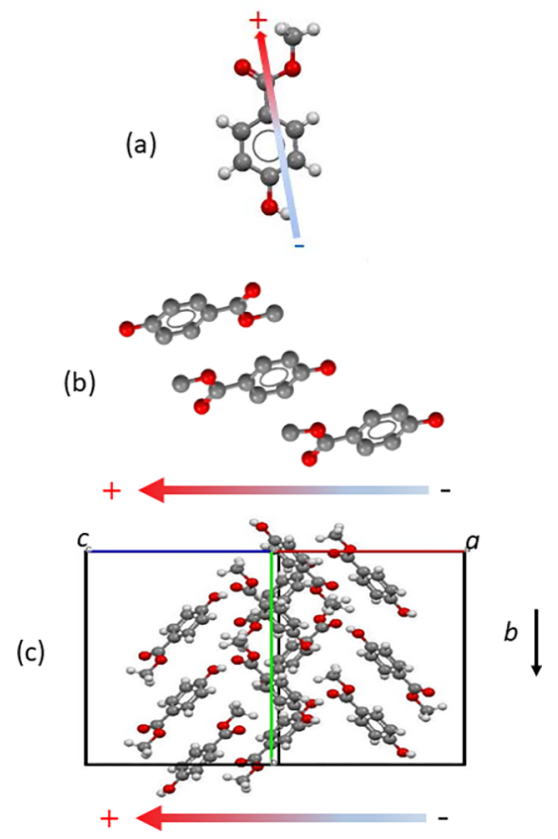

Figure 1. (a) The molecular structure of MHB showing the orientation and sign of the molecular dipole. (b) The asymmetric unit, showing the 2:1 ratio of polar directions in the triplet of molecules and the total polar direction. (c) The crystallographic structure of MHB as viewed perpendicular to the (101) plane, indicating the total polar direction.

\section{EXPERIMENTAL SECTION}

Materials. MHB (Sigma Chemical Co., 99.04\%), greyish-white in color, was purified by recrystallization, continuous column chromatography, and sublimation. ${ }^{34}$ The purified products were monitored by high-performance liquid chromatography (HPLC) using a Shandon Scientific Hypersil CTA column. The HPLC showed the presence of three impurities (unidentified) in the as-received material; two of them could be removed by solution purification methods (recrystallization and chromatography), and all of them could be removed by gradient sublimation. The purity of the product after sublimation rose to $99.95 \%$, which was pure enough to use for crystal growth both from solution and by PVT.

MHB exhibits a complex polymorphism. Early experiments using optical microscopy indicated the potential existence of at least six polymorphic forms, of which two were definitely identified. In recent years following the increased interest in polymorphism and polymorphic relationships in organic materials, a number of X-ray crystallographic studies have been performed on MHB. These have confirmed the existence of the above two forms and identified several of the other potential forms. We do not propose to review these studies, details of which are adequately reported in the papers referenced and their additional supporting evidence. ${ }^{35-38}$ Germane to the present problem however is that a number of these later rediscovered polymorphs were prepared following sublimation of the roomtemperature stable form. Consequently, we must state that all of the material purchased, purified, and grown into crystals under the experimental conditions used in the present experiment and the resulting single crystals were defined by differential scanning calorimetry (DSC) and X-ray diffraction (XRD) to be of the roomtemperature stable polymorph. All showed no evidence of any polymorphic transformations before a sharp melting temperature of $124{ }^{\circ} \mathrm{C}$.

Saturation vapor pressures (SVP) for MHB were assessed by extrapolation of the recently published data of Perlovich et al. ${ }^{39}$ Using a dynamic assessment technique in the temperature range from 30 to 54 ${ }^{\circ} \mathrm{C}$, these authors showed that the SVP follows a relationship

$$
\ln (P[\mathrm{~Pa}])=(34.3 \pm 0.3)-(11889 \pm 92) / T
$$


In the PVT experiments supersaturations are given as $\sigma=\left(p_{\mathrm{o}}-p\right) / p_{\mathrm{o}}$, where $p$ is the vapor pressure at the deposition temperature and $p_{\mathrm{o}}$ that at the source temperature.

2.3. X-ray Crystallography. Single-crystal X-ray diffraction studies performed at $100 \mathrm{~K}$ on samples prepared from the above material yielded results that were in excellent agreement with other similar assessments. ${ }^{35-38}$ The results of our examination confirmed that the structure of MHB solved as monoclinic with $a=13.360 \AA, b=17.087 \AA$, $c=10.958 \AA, \beta=119.87^{\circ}, Z=12$, and space group $C c$, equivalent to the nonstandard setting as given by Lin. ${ }^{35}$ The calculated Flack parameter defined the absolute configuration, and as the crystal was of known provenance, being extracted from a larger single crystal (exhibiting growing and nongrowing facets), the relationship between the absolute configuration and growing/nongrowing faces can be determined. For continuity and consistency with previous studies, we base our discussion on the nonstandard setting used by earlier authors. ${ }^{35,38}$

A full analysis of the absolute configuration of the structure will be reported in full in a future publication. ${ }^{40}$ Figure $1 \mathrm{~b}$ shows the asymmetric unit of $\mathrm{MHB}$ and the resultant polar direction, and $1 \mathrm{c}$ shows the unit cell of the crystal as viewed perpendicular to the (101) plane, showing that eight of the 12 molecules orientated with the methoxyl groups (positive ends) pointing in the general [001] direction compared with four pointing in the general $[00 \overline{1}]$ direction. The polarity of the asymmetric unit is retained by the c-glide plane. There are no centers of inversion to negate the overall polarity of the asymmetric unit when building to the unit cell. Overall, this will mean that the (111) and ( $\overline{11} 1)$ growth faces will be predominantly populated by the methoxyl groups and present an electrically positive character and that the opposite faces, (111) and (111) predominantly populated by hydroxyl groups, will present a general negative character.

$\mathrm{X}$-ray section and projection topographs ${ }^{41,42}$ of selected crystals were recorded at the Daresbury, UK, Synchrotron Radiation Laboratory using the white radiation source at station 7.6. The spectral range of the radiation was $0.5-2.5 \AA$, and the selected experimental wavelength was $1.54 \AA$ Å.

Optical Microscopy was performed by direct interference and interference contrast techniques using a Leitz-Reichart Polyvar 2 microscope.

Theoretical Modeling of the Equilibrium Morphology of MHB and Polarity Calculations. For comparison with the PVT grown crystals, a theoretical model for the equilibrium crystal morphology of MHB was determined using the program HABIT 95. ${ }^{43}$ With the measured crystallographic data, attachment energies $\left(E_{\text {att }}\right)$ were calculated using a Dreiding II force field combining a Lennard-Jones 12-6 potential and a 10-12 hydrogen bond potential with parameters from Mayo, Olafson, and Goddard. ${ }^{44}$ The results of the calculation are presented and discussed below. Polarity calculations were performed using MOPAC2016, using an AM1 basis set.

\section{CRYSTAL GROWTH EQUIPMENT}

Gradient Sublimation. An essential preliminary to PVT growth is the definition of the conditions of temperature and supersaturation likely to produce individual nuclei that will grow to yield separated crystals of good and well-defined habit and hence, high perfection. We found it useful to define these conditions by the use of a temperature gradient sublimation equipment of the type described by McArdle and Sherwood. ${ }^{34}$ With this equipment, the influence of the potential factors that might be of influence in the growth process, namely, temperature, ambient pressure, and hence supersaturation, can be performed speedily and accurately. Details of the procedure and results for the present material are given in the attached electronic Supporting Information Figure S1.

Crystal Growth at Defined Supersaturations. The main crystal growth equipment (electronic Supporting Information S2) was modeled on the technique developed by Piper and Polich ${ }^{45}$ and adapted for organic crystal growth by Sherwood et al. $^{34}$
The supersaturation in the growth zone was calculated from the equilibrium vapor pressures estimated for the growth and source temperatures and is referred to as the Nucleation Supersaturation for reasons that will become apparent as the investigation proceeds. Most of the crystals grown at this time were initiated by self-nucleation. A preliminary investigation of seeded growth was also attempted initially by inserting small crystals mounted on polymer film into the upper growth chamber before growth was commenced.

A later attempt at controlled seeded growth of larger crystals was made using an adaptation of the Catano and Kun ${ }^{46}$ technique for the production of inorganic semiconductor crystals. This involved a reconfiguration of the electrical windings in the growth equipment to yield a temperature gradient in the growth furnace and a redesigned growth ampule the form of which will be described at a later point in the text (electronic Supporting Information S3).

\section{RESULTS AND DISCUSSION}

Structural Considerations. Table 1 shows the Habit 95 calculation values for the attraction energies $\left|E_{\text {att }}\right|$ on the various potential growth faces of MHB:

Table 1. Attachment Energies $\left(E_{\text {att }}\right)$ Calculated for MHB using the Habit 95 Program

$\begin{array}{lccccc}\text { crystal face } & \{11 \overline{1}\} & \{110\} & \{020\} & \{021\} & \begin{array}{l}\{202\},\{111\} .\{200\}, \\ \{002\}\end{array} \\ & & & & & \leq-28.54 \\ E_{\text {att }} / \mathrm{kJ} \mathrm{mol} & -13.59 & -13.75 & -22.53 & -28.51 \mathrm{~s} & \leq-28.5\end{array}$

Faces with the lowest values of $\left|E_{\text {att }}\right|$ are those most likely to form the largest habit faces of the crystal. Those showing lower I $E_{\text {att }} I$ values will be successively less important. In this event the predicted dominant morphology will be $\{110\}$, (11 $\overline{1})$ with $\{020\},\{021\}$ as potential smaller faces as shown in Figure 2.

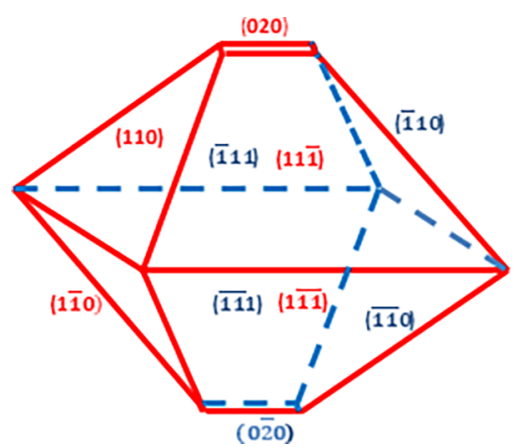

Figure 2. Equilibrium morphology of MHB calculated from the data given in Table 1.

With the exception of one very striking occurrence, faces with attachment energies lower than the above were rarely observed. This exception was the presence of the $\{002\}$ form under some conditions of growth.

In describing these features, please note that the experimentally found $(h 00),(0 k 0)$, and (00l) faces will have $h=k=l=$ \pm 1 ; however in all modeling programs, these are returned as $h=$ $k=l= \pm 2$, following the systematic absences found in diffraction (i.e., the $h=k=l= \pm 1$ do exist, but the habit calculation programs treat them as if they do not). We followed the 
modeling convention in labeling these faces for consistency's sake.

It must be recalled that the HABIT 95 calculation takes no account of the acentric nature of the MHB lattice and thus predicts an equivalent habit for the faces propagating in both the polar directions $[001]$ and $[00 \overline{1}]$. In an acentric material this will not be the case, and it is well-known that, for some such materials, the morphology and growth behavior of the opposite polar faces may differ considerably. While growth in one polar direction is normal, growth in the opposite direction is zero or, at best, very slow. Reference to Figure 1c and the accompanying text confirms that the crystal faces propagating with a growth vector in the general [001] direction will exhibit an electrically positive character and that those propagating in the opposite direction a negative character; that is, the (111), ( $\overline{111})$, and (021) faces will exhibit a positive character, while $(11 \overline{1}),(1 \overline{11})$, and $(12 \overline{1})$ faces will exhibit a negative character. This relationship for $\mathrm{MHB}$ was confirmed by assessing the polarity of the pyroelectric current developed on heating a morphologically well-defined crystal and from the absolute configuration studies described above. ${ }^{40}$ This indicated that the fast-growing faces were negative and that the slow and even possibly zero growth faces were positive. Experience with a number of related acentric highly polar materials ${ }^{10,22,23,47-49}$ leads us to expect that the negative leading faces will grow normally and exhibit visually flat and well-formed growth facets. These match well with the predicted forms. In contrast the latter (positive leading) show little or no growth and may show elements of the predicted shape, but they will be rough, ill-formed, and rounded.

Crystal Growth Experiments. Temperature gradient tests indicated that the most promising conditions for the crystal growth of MHB lay in the region source temperature 120 ${ }^{\circ} \mathrm{C}$, deposition temperature $80-90{ }^{\circ} \mathrm{C}$, and operation in vacuo. (electronic Supporting Information S1).

Single Crystal Growth. Growth by Self-Nucleation. Following the insertion of the ampule into the PVT growth system (electronic Supporting Information, Figure S2) a negative temperature differential was established with the source region at $90^{\circ} \mathrm{C}$ and the region of deposition at $120^{\circ} \mathrm{C}$. This served to clean the ampule wall of any powdered material following preparation. After $1 \mathrm{~h}$ the gradient was reversed to give the defined growth conditions: source temperature of $120{ }^{\circ} \mathrm{C}$ and deposition fixed at a definite temperature in the promising range.

Under these conditions nucleation usually occurred after $\sim 10$ to $12 \mathrm{~h}$ to give 3-6 nuclei within the growth region. Limitation of further nucleation and promotion of subsequent growth could be then finely controlled by adjusting the temperature in the upper portion of the growth system.

In this manner, prismatic crystals can be grown in sizes up to several centimeter dimensions in periods of 7-10 d. Figure 3 shows typical crystals grown by PVT under a range of supersaturations. Overall the crystals showed the same basic forms predicted by the HABIT 95 calculations. A minor distinction in detail is that, while the Habit 95 calculation predicts larger $(11 \overline{1})$ and $(1 \overline{11})$ faces than $\{110\}$ faces, the experimental crystals showed the reverse with $\{110\}$ faces elongated along the $[00 \overline{1}]$ direction. $(11 \overline{1})$ and $(1 \overline{11})$ faces, small and infrequent at the lowest supersaturations, became more prominent with increasing supersaturation. (02 $\overline{1})$ and $\{020\}$ were seen frequently but were always much less dominant than the two major forms at lower to medium supersaturations. The $(02 \overline{1})$ faces increased in dominance as the supersaturation (a)
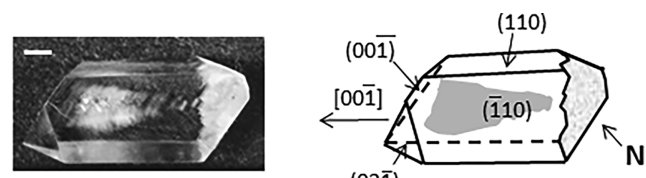

(02א)

(b)
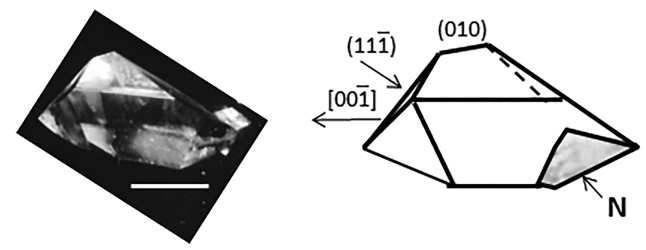

(c)
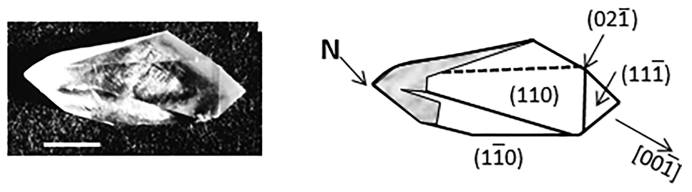

(d)
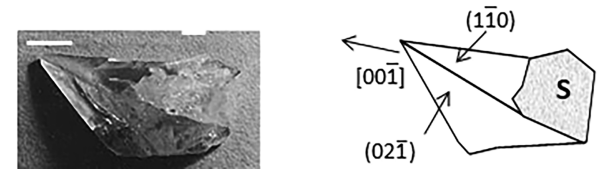

Figure 3. Crystals of MHB grown by PVT. Self-nucleation at supersaturations of (a) 0.92, (b) 0.96, (c) 0.98. (d) Seeded growth, supersaturation 0.92 . (N) Nucleation point. (S) Original position of seed. The nonpropagating surfaces areas are shaded (scale mark $1 \mathrm{~mm}$ in all cases).

increased. Surprisingly an unexpected $(00 \overline{1})$ form played a persistent role in the crystal development and perfection particularly at lower supersaturations.

In the crystal shown in Figure 3a $(\sigma=0.92)$ the $[00 \overline{1}]$ growth direction is terminated by a large $(00 \overline{1})$ face and smaller $(02 \overline{1})$ type face. In all cases in which a dominant $(00 \overline{1})$ face was found it was preceded by a visible cone of imperfection in the crystal. More perfect crystals grown at intermediate supersaturations $(\sigma$ $=0.96$, Figure $3 \mathrm{~b}$ ) developed to yield a bipyramidal habit that showed no $(00 \overline{1})$ face and were bounded by large $\{110\},(11 \overline{1})$, $(1 \overline{11})$, small $\{010\}$, and occasionally $(02 \overline{1})$ faces. A second larger example of this type of crystal was used for the section topography shown in Figure 6. The morphology, shown diagrammatically in Figure $6 a$, can be confirmed from the shapes of the X-ray section topographic images presented therein. Many of the crystals in this supersaturation region,

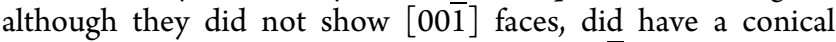
volume of inclusions localized along the $[00 \overline{1}]$ direction (see Figure 6). The reason for this will become apparent in the next sections and discussed more fully after a discussion of Figure 6.

Moving to higher supersaturations (Figure $3 c, \sigma=0.98$ ) resulted in the development of all predicted growth faces (11) $)$, $(1 \overline{11}),(02 \overline{1})$, and $\{110\}$ with the increasing dimensions of the first two forms leading to a skewing of the habit along [00프. In parallel the optical quality of the crystals decreased due to the formation of obvious inclusions and strain-induced cracks in all sectors during growth.

At higher supersaturations $(\sigma=0.99)$ the $(11 \overline{1})$ and $(1 \overline{11})$ faces had also "grown out", and the crystals produced comprised only $\{110\}$ and $(02 \overline{1})$ faces. They were of similar morphology and imperfection to that shown for seeded growth in Figure $3 \mathrm{~d}$.

Attempts were made to accelerate the rate of growth of the crystals once satisfactory growth had been initiated. Increasing the temperature differential to source $120{ }^{\circ} \mathrm{C}$ and growing crystal to $75^{\circ} \mathrm{C}(\sigma=0.99)$ resulted in the further development of 
both the habit and a deterioration in the visual perfection of the growing crystals. Although they continued the morphological development shown in Figure 3 to a $(11 \overline{1}),(1 \overline{11}),\{110\},(02 \overline{1})$ habit with $\{110\}$ increasingly predominant, the crystals lost their transparency due to an increased formation of defects in the bulk.

Seeded Growth. In an attempt to restrict the number of nuclei and hence growing crystals and to set a better-defined supersaturation for growth, small, perfect, seed crystals of several orientations were attached to polymer film. These were inserted into the growth ampule to lie in the temperature range of 90-95 ${ }^{\circ} \mathrm{C}$ (supersaturation $\sigma=0.92-0.98$ ), which is the range of satisfactory growth indicated by our self-nucleation experiments.

The experiment was successful in that many seed crystals grew at rates relative to the supersaturation to which they were exposed. Some seed crystals however did not grow. These potentially were seed crystals that, by chance, were oriented such that the positive $c$-direction faces, that is, the potential nongrowing direction, were exposed to the vapor source. An example of a seeded growth is shown in Figure 3d. Being set at experimental conditions corresponding to a nucleation supersaturation of $\sigma \approx 0.92$ to 0.96 the crystal was expected to yield a columnar or octahedral morphology similar to that shown in Figure $3 \mathrm{a}$ or $3 \mathrm{~b}$. Instead, the resulting morphology was more characteristic of growth at a higher supersaturation $(\sigma>0.98)$.

These observations pose two questions.

- Was seeding yielding different growth characteristics of the growth process in the negative growth direction?

- Was the lack of growth in the positive $c$ direction a characteristic feature of the growth of this material, a feature not recognized in all previous growth studies?

These questions were tested in two experiments outlined below.

The role of supersaturation in defining the differences between self-nucleated and seeded growth was defined by a step toward the growth of larger crystals using a system based on the previous studies of Catano and $\mathrm{Kun}^{46}$ on the PVT growth of larger crystals of inorganic materials. This required a reconfiguration of the furnace windings depicted in electronic Supporting Information 2 to yield a temperature gradient and a redesigned growth ampule, both of which are also depicted in the electronic Supporting Information S3.

A typical result obtained is shown in Figure 4. A crystal seed set to grow at the basic predicted supersaturation of $T_{s}=120^{\circ} \mathrm{C}$, $T_{\mathrm{g}}=115^{\circ} \mathrm{C}, \sigma=0.92$ and hence to form a columnar crystal (type
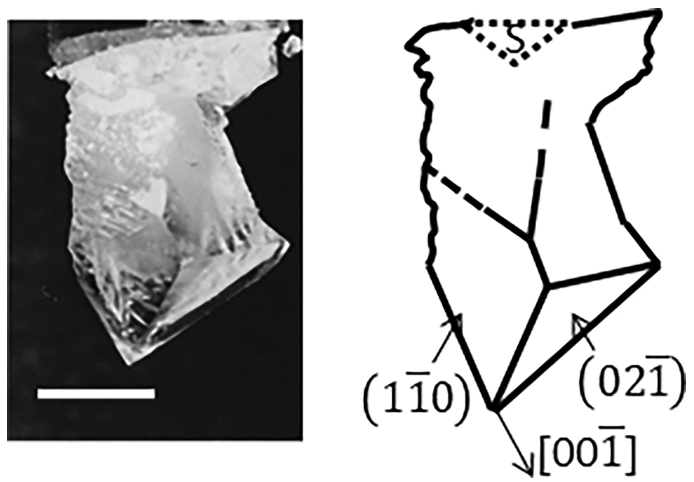

Figure 4. A typical crystal grown by the Catano and Kun technique at a growth supersaturation of $\sigma=0.92 . \mathrm{S}=$ Seed.
Figure 3a), transforms into the predicted $\{110\},(02 \overline{1})$ morphology characteristic of growth at relatively high supersaturations $\sigma>0.98$. It also replicates the overall shape of the crystal resulting from the trial seeding experiment performed during the course of the general study of self-nucleation and under the same growth conditions. The fact that the seed crystal, set to grow from well-defined $(11 \overline{1})$ and $(1 \overline{11})$ faces, transforms to yield $(02 \overline{1})\{110\}$ growth underscores the dominant role that supersaturation plays in defining the morphology of a growing crystal and questions the true identification of the growth supersaturation. "Is there a major distinction between the nucleation supersaturation and that which is active during subsequent growth even though the initial nucleation supersaturation is apparently retained?". We answer this question in the conclusions section.

Regarding the question of whether or not there is no growth on the positive leading faces of this material we present further definitive evidence. With a growth cell designed to study in detail the kinetics of the growth of the title material and which will be described in full in a future publication, ${ }^{47}$ examinations were made of the early stages of growth of MHB. Figures 5 and 6

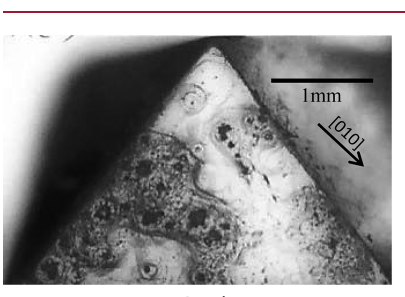

$0 \mathrm{~min}$.

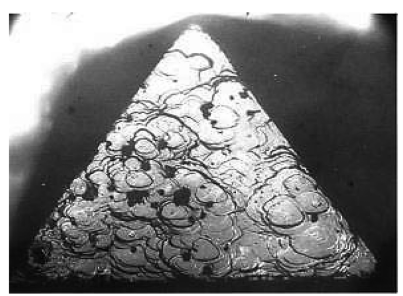

$100 \mathrm{~min}$.

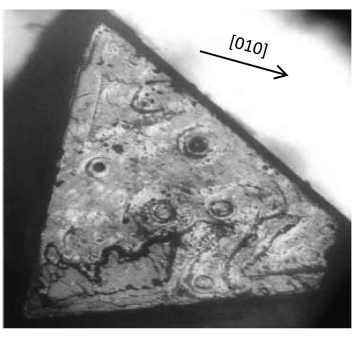

$0 \mathrm{~min}$

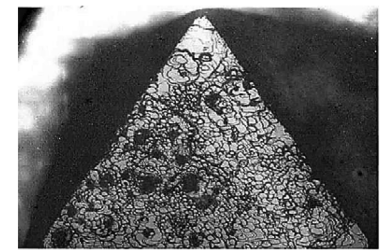

$10 \mathrm{~min}$.

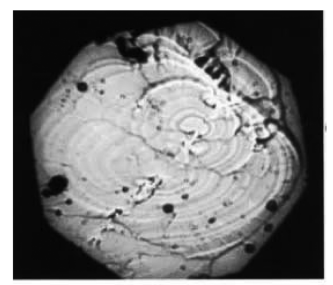

$100 \min .16 x$
(11) Face

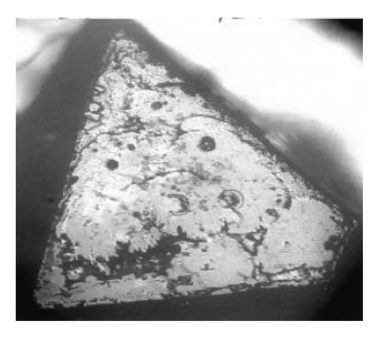

(111) Face

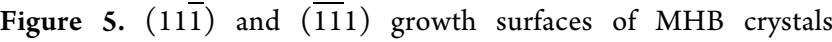
photographed in situ during growth at the indicated times. Supersaturation $\sigma=0.32$. The $16 \times$ enlargement provides detail of an area of the $100 \mathrm{~min}$ photograph showing the structure of the growth hillocks.

depict the development of surface features during the early stages of regeneration of the crystal surfaces. The photographs at $t=0$ shows the initial full crystal surfaces of a seed crystal prepared by growth from methanol solution. These are roughened by the etching that results inevitably on removal and drying of the seed crystals from the growth solution. Growth conditions in each case were set at source temperature $T_{\mathrm{s}}=120$ 


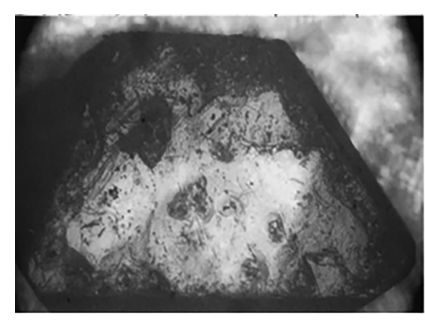

$0 \mathrm{~min}$.

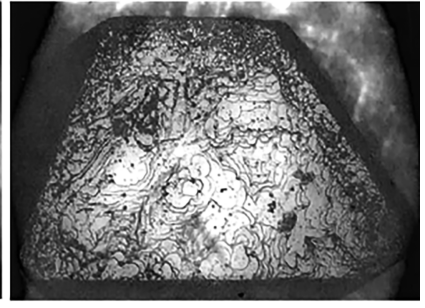

$30 \mathrm{~min}$.
$\{110\}$ Face

Figure 6. $\{110\}$ growth surface of an MHB crystal photographed in situ during development at the indicated times. Supersaturation $\sigma=0.32$.

${ }^{\circ} \mathrm{C}$ (growth temperature, $T_{\mathrm{g}}=115^{\circ} \mathrm{C}, \sigma=0.32$ ), well below the projected nucleation supersaturation range of the self-nucleation experiments. Within a short period $(10$ and $30 \mathrm{~min}$, respectively) growth on potentially dislocation nucleated growth centers is well-established on both the (110) face and the negative $(11 \overline{1})$ face. That the growth features are elongated hillocks is not discernible at this magnification, but enlargements at later times (depicted for the (111) face in Figure 4) confirm this structure. The shape and orientation of the growth centers match well with the underlying crystallographic surface structure and face shape, allowing for distortion caused by the unequal crystallographic axes. They are roughly distorted triangular on the (11i) faces and trapezoidal on the $\{110\}$ faces. The long $(\mathrm{L})$ and short $(S)$ axes lie along: (110) faces, $L,[1 \overline{1} 2]$ and $S,[1 \overline{11}]$ directions and $(11 \overline{1})$ faces, $L[1 \overline{1} 0]$ and $S$ at right angles to this. These growth centers continued to develop with increasing time to form well-defined spiral growth centers. ${ }^{47}$

In contrast with the early development of growth centers on the $(110)$ and $(11 \overline{1})$ faces, even after $1.7 \mathrm{~h}$ no growth centers have formed on the $(\overline{11} 1)$ face. This inactivity continued to longer times thus confirming absolutely a complete absence of growth on $(\overline{1} 11)$ and $(\overline{11} 1)$ faces propagating in the general $+c$ direction of growth under what can be regarded as normal supersaturation conditions

Crystal Perfection. Figure 7 shows a series of synchrotron Xray section topographs of the crystal shown in Figure 3a that was grown at $\sigma=0.92$. Three (001) sections representing different growth stages marked 1,2 , and 3 were investigated. The images shown were obtained from (002) reflections with a Bragg angle of $\sim 15.8^{\circ}$ at a wavelength of $1.54 \AA$.

Figure 7 immediately defines that the visible conical imperfection in the crystal depicted in Figure 3a is contained in a developing, and defective, (001) growth sector. Rectangular images (annotated I) of this sector, increasing in size as growth progresses, appear at the center of all three section topographs. These back extrapolate to an apex that lies close to the [001] extremity of the crystal. This confirms that the crystal has nucleated at a point close to or coincident with the [001] extremity of the crystal and has developed predominantly in the [001] direction. As noted above the rough appearance of the faces with growth vectors pointing in the [001] direction is characteristic of the nongrowing faces of these materials in general. ${ }^{10,22,23,48-50}$

The topographic images reproduced are of a quality characteristic of a good solution-grown crystal. The lateral growth sectors are the $\{110\}$ family of crystal forms. Image (1), closest to the nucleation point, shows good-quality crystal in all these sectors. The central sector (I) and its shape clearly represents a developing [001] growth sector.
(3) (2) (1)

(1)

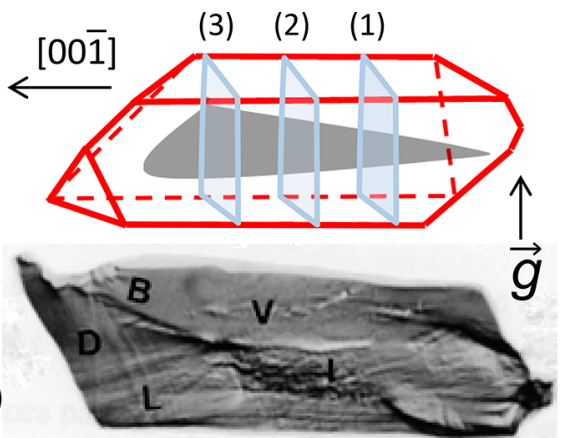

(2)

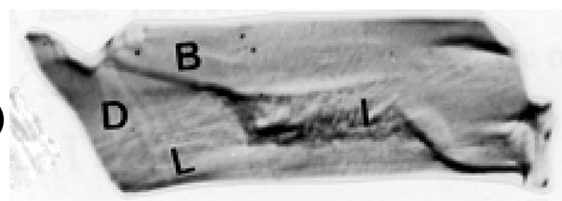

(3)

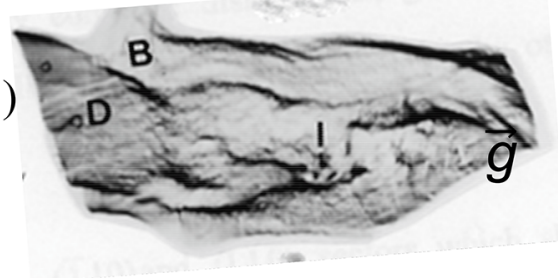

Figure 7. X-ray section topographs of a crystal of the type shown in Figure 3a showing the imperfection localized and developing in the $\{00 \overline{1}\}$ sector. For annotations see text.

The images of dislocations (D) extending from the central region normal and near normal to the exterior growth faces are

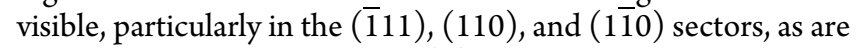
the growth sector boundaries (B) between these sectors. The occurrences of growth inclusions (V), growth layers (L) in equivalent positions in the same sectors, are consequences of fluctuations in growth rate due to variations in temperature and flux during the growth experiment.

Figure 8 presents a similar analysis of a crystal grown at the higher supersaturation of $\sigma=0.96$. Crystals grown in this supersaturation region show no $(00 \overline{1})$ faces, and the $\{11 \overline{1}\}$ and (1) faces have taken over the lead in the $[00 \overline{1}]$ growth of the crystal.

To these faces are often added small $\{010\}$ faces and, less frequently, $(02 \overline{1})$ faces. The topographs presented in the figure

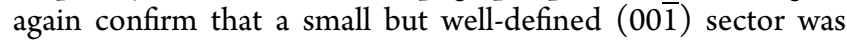
formed early in the development of the crystal. This is the rectangular feature at the center of the topograph, Figure 8a, which is closest to the nucleation point of the crystal. If this face were to continue to propagate, then it should also be seen as a well-defined, larger, rectangle of growth sector boundaries at the center of the topograph Figure $8 b$, which was further from nucleation. Despite the unfortunate formation of a crack line across the center of the image, obviously such an image is not present. Its initial presence however continues to be indicated by the inclusions along the $[00 \overline{1}]$ direction noted above. Thus, the fast growing $(00 \overline{1})$ growth sector, so dominant in growth at low supersaturation, has been overtaken and eliminated by slower developing $\{110\}$ and $(11 \overline{1})$ growth sectors. With the elimination of this sector the overall perfection of the developing crystal has improved. Similar defect features to those defined in Figure 7 are visible on the topographs. The clarity of the 


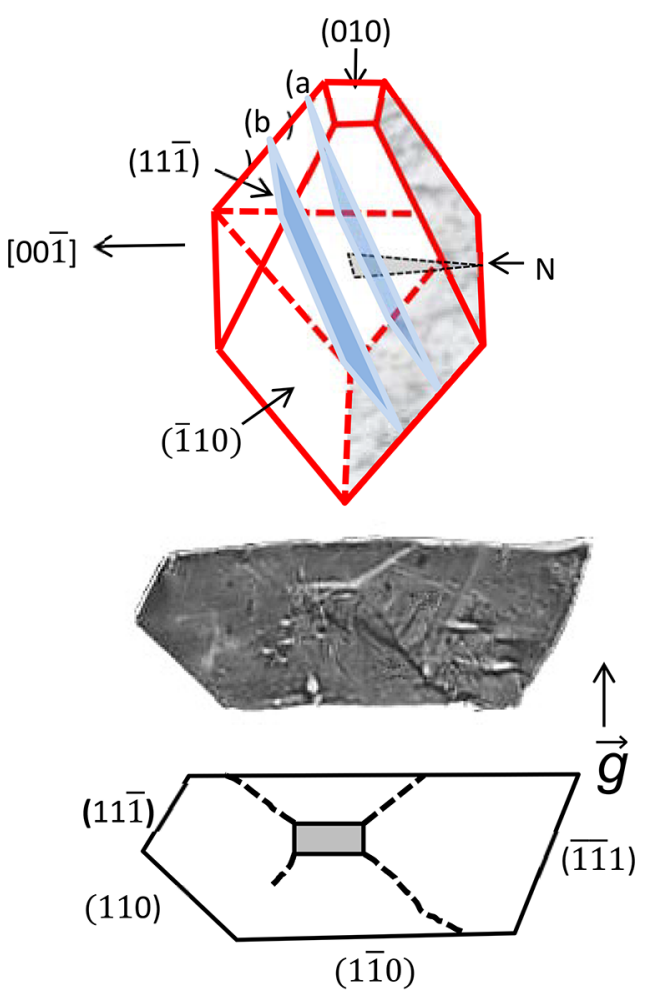

(a)

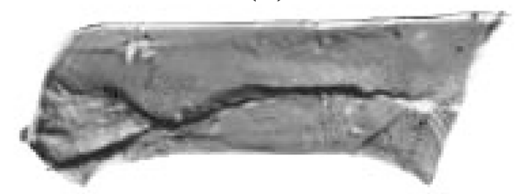

(b)

Figure 8. X-ray (001) section topographs of a bipyramidal crystal grown at $\sigma=0.96$ showing the gradual loss of the 001) sector, visible in (a) but absent in (b) and leading to an improvement in perfection of the surrounding growth sectors. Crystal size $=1.5 \mathrm{~cm}$.

topographs and their defect features is testament to the generally high structural perfection of the crystal.

Morphological Development. From a wider range of experiments and observations we can define the morphological development of MHB with increasing supersaturations. Table 2 summarizes this series of events.

At supersaturations less than ca. 0.88 and following nucleation, the growth rates of the various faces are immeasurably low, the growing crystallites are small, and we have no reliable data. At approximately this supersaturation the principle habit faces have formed, and the future pattern of development becomes apparent. The growing crystal takes up the columnar form with dominant $\{110\}$ faces and, surprisingly, a large, unpredicted, $(00 \overline{1})$ face. The $(11 \overline{1})$ and $(1 \overline{11})$ faces are nonexistent. The continued existence of the (001) face is stabilized by the development of considerable defect structure in the $(00 \overline{1})$ sector as it advances. In some cases where this imperfection is significant the sector and face can continue to propagate and dominate over the more favored $\{11 \overline{1}\}$ faces to even higher supersaturations. Under normal circumstances however the consequential higher growth rate of this face causes it to "grow out". Growth in the [001] direction is gradually taken over by the advancing $(11 \overline{1})$ and $(1 \overline{11})$ faces. The initial presence of the $(00 \overline{1})$ sector is generally marked however by a residual cone of inclusions lying along the $[00 \overline{1}]$ axis of even the most perfect MHB crystals. This feature is also apparent in crystals of MHB grown from solution as will be seen in an accompanying manuscript on this topic. ${ }^{51}$

From this point the rate of growth of the $(11 \overline{1})$ faces propagating in the $[00 \overline{1}]$ direction increases. They are joined by the increasing presence of smaller, less morphologically important, $\{010\}$ and $(02 \overline{1})$ type faces. In parallel with the increasing dominance of these faces a gradually increasing degree of structural imperfection develops in both the rapidly advancing sectors propagating in the [001] direction and in the lateral $\{110\}$ sectors. At all times the end of the crystals facing in the [001] direction showed little or no growth.

A final consideration is to define why the observed variations in morphology occur. We can only conclude that they reflect a variation in the mechanism of growth in the various stages of supersaturation dependence.

Micromorphology of the MHB Habit Faces. It is well-known that the growth mechanism of a crystal can vary with supersaturation. $^{52}$ Once growth steps have been generated from the source, whether $2-\mathrm{d}$ nuclei or dislocation outcrops, their velocity, heights, and shape will vary with the supersaturation. The dislocation activity can also change with supersaturation and provide additional sources for the steps. The incorporation mechanism can also change. At low supersaturations, singly adsorbed molecules are most likely to be incorporated at the kink sites available on the steps. At high supersaturations, the concentration of the adsorbed molecules increases, and it will be easier to form 2-d islands. In extreme cases, the driving force is so high that adsorbed molecules do not need to find the kinks and sinks to form the crystal lattice. Every site on the surface becomes active to the incorporation of molecules, and a rough growth mechanism initiates. It can be helpful to understand these variations in the light of growing large and perfect optical crystals and, hence, to achieve the minimization of imperfections in as-grown crystals. Information on such changes and their influence can be obtained from examination of the micromorphology of the growing crystal surfaces.

Figure 9 presents the variation of the micromorphology of the growth faces of $\mathrm{MHB}$ as a function of supersaturation. The $\{110\}$ faces were the slowest growing faces in the crystal. They yielded surface features that were stable and easy to observe. They also form the major growth sectors in the crystal and hence make a major contribution to the volume of usable material following growth.

At a supersaturation of $\sigma=0.88$ (Figure 9a), the growth sources are elongated roughly shaped growth hillocks with strong orientation. The steps generated are usually low, and the size of the growth hillocks varies from 10 to $15 \mu \mathrm{m}$ in length and $\sim 5-8 \mu \mathrm{m}$ in width. This indicates a strongly anisotropic growth along the long and short axis direction of the growth hillock.

When the supersaturation increased to $\sigma=0.93$ (Figure 9b), the micromorphology of the growth hillock changes from an elongated ellipse to a pyramid. On the top of the pyramid, the outcrops of dislocations could be seen clearly. Such a change in the shape and distribution of growth hillocks indicates that the anisotropy of step velocity in the surface becomes reduced. In other words, the relative step velocity on the (110) faces changes with an increase of supersaturation. Meanwhile, the height of the steps is still similar to that observed at a supersaturation of 0.88 .

Further increase in supersaturation $(\sigma=0.96)$ (Figure 9c) produces no further change in the shape of the growth hillocks. 
Table 2. Morphological Variations with Supersaturation of Crystals of MHB Grown by Self-Nucleation from the Vapour Phase

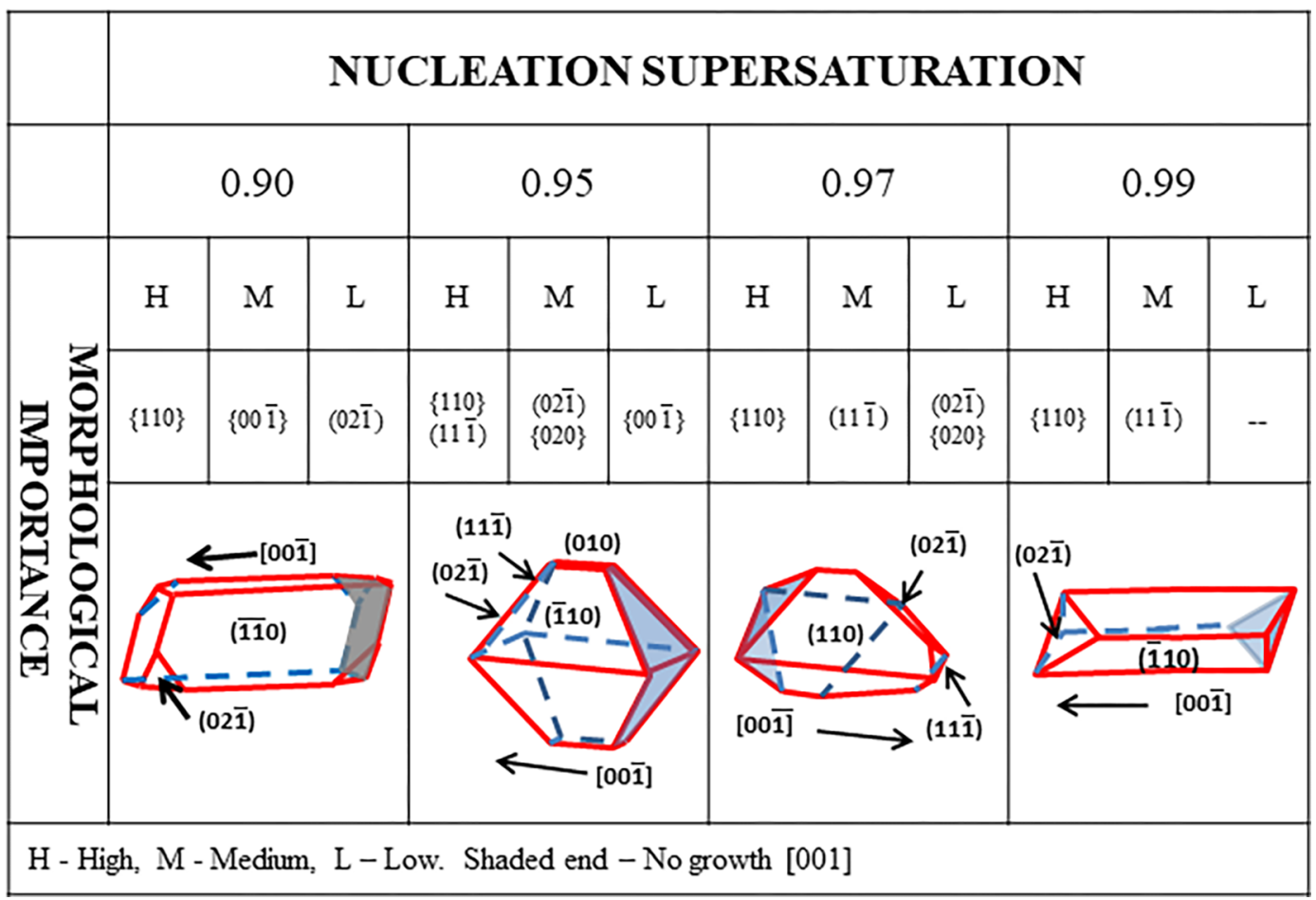

\section{$\{110\}$ FACE}
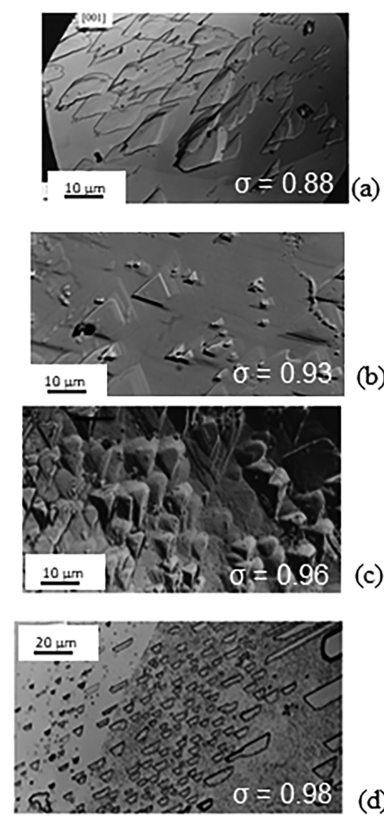

(c)

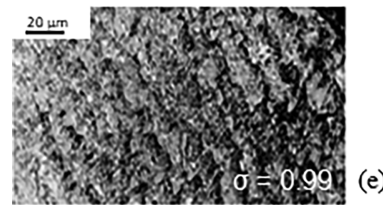

(d)

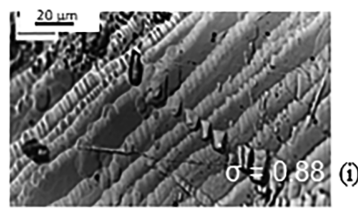

Figure 9. Surface micromorphology of the various growth faces of $\mathrm{MHB}$ as a function of supersaturation $(\sigma)$.

These are still pyramidal. What does change is the slope of the vicinal faces of the hillocks and the height of steps generated. The former become steeper, and the latter become higher. Close examination reveals several flat top growth features. These are 2dimensional (2-d) nuclei scattered among the growth hillocks. Thus, a combination growth mechanism, BCF $+2-\mathrm{d}(\mathrm{BCF}=$ Burton, Cabrera, and Frank), commences, with the former mechanism dominant.

At still higher supersaturations $(\sigma=0.98)$, (Figure 9d), numerous 2 -d islands become apparent on the $\{110\}$. The 2 -d islands have the same elongated pyramid pattern as the growth hillocks noted at lower values of supersaturation. This is a good example of a 2-d growth mechanism. The different size of islands indicates that they exist in different growth stages. An interferometric image clearly defined their flatness. When such a growth mechanism starts, the MHB crystal develops a prismatic structure with dominant (110) faces.

Finally, at $\sigma=0.99$ the (110) surfaces develop a roughness. In such a circumstance, no further anisotropy of the growth step velocity can be distinguished. Every site on this surface is so active that all adsorbed molecules are incorporated immediately. The growth rate then accelerates. Without any doubt, such a growth mechanism will only produce crystals of poor optical quality with large volumes of inclusions.

Similar observations of the micromorphology of the (11) faces propagating in the faster growing [00] direction showed them to follow a similar pattern of behavior. At low supersaturations the $(11 \overline{1})$ faces of $\mathrm{p}-\mathrm{MHB}$ grew by a dislocation-controlled mechanism. With an increase in supersaturation this changed successively to a combination (BCF and 2-d) mechanism and 2D mechanism. Finally, at the highest supersaturation, the surface roughens.

This parallel behavior of the two principal faces of the growing crystal fits well with the observed structural features noted in the growing crystals; in particular, the manner in which inclusions and imperfections develop in the various sectors. Obviously the best growth occurs in supersaturation ranges in which controlled growth by a dislocation mechanism dominates. The gradual 
onset of 2 -d growth, first in the $(11 \overline{1})$ sectors and eventually in the $\{110\}$, signals the onset of more rapid and defective growth in the crystal as a whole. It is interesting to note that this deterioration is exacerbated by the formation of larger $(02 \overline{1})$ faces at later stages of the supersaturation range. As Figure $9 \mathrm{i}$ shows, the surface structure of these faces is very heavily faulted with gross macrostep formation. The role of steps of increasing height in inclusion formation and defective growth is welldocumented both experimentally and theoretically. ${ }^{53}$

In contrast to the series of developments on the $\{110\}$ and $(11 \overline{1})$ faces the nongrowing faces showed a general rough and eroded surface compatible with the general rough visual appearance.

\section{CONCLUSIONS}

Unidirectional Growth. The morphology of crystals of MHB grown from the vapor phase follow the general pattern of unidirectional growth that has become expected of this class of structurally acentric solids. While faces that propagate with a growth vector in the electrically positive, $+c$ direction show little or no growth, those that face in the negative direction grow normally. In the latter direction the morphology is dominated by faces defined as the most probable by theoretical morphological calculations, namely, $\{110\},(11 \overline{1}),(1 \overline{11}),(02 \overline{1})$, and $\{020\}$. Their relative sizes result in a gradation of shapes varying from columnar to skewed bipyramidal, to elongated bipyramidal and, finally, to needlelike. This variation coupled with the parallel gradually increasing imperfection of the crystals is shown to result from changes in the controlling growth mechanism with applied supersaturation, as evidenced by the micromorphology of the crystal faces. Not surprisingly, the highest perfection is found in crystals grown in a supersaturation regime in which growth is controlled by a dislocation nucleated mechanism. This is also the region in which the crystal morphology is most similar to that predicted by theoretical (HABIT 95) calculations. This observation leads to the obvious conclusion that scale up to growing crystals of good quality by PVT processes requires that these essential conditions are met. This conclusion will hold whether the crystals being grown are of large $(\mathrm{cm})$ or very small (sub-mm) sizes.

Why the crystals fail to grow in both polar directions is still an open question. Various theoretical models have been put forward to explain this unidirectional behavior for the material $\alpha$-resorcinol. ${ }^{24-27}$ These models relate specifically to the case of that material and how its molecules crystallize at the crystal surfaces. In very general terms these models indicate a higher degree of disorder at the nongrowing surface than at the growing surface. As experimentalists our minds focus on the mechanistic changes that take place.

Both the positive and negative faces show very little if any growth at very low supersaturations. At some critical supersaturation the negative faces become active and propagate by a dislocation controlled (BCF) mechanism. This is rational and is covered by existing theory. ${ }^{54}$ Any pre-existing dislocations will remain inactive until the radius of curvature of the growth spiral generated by the dislocation exceeds a critical radius; then growth accelerates. It may well be that the theoretically proposed disorder at the positive face discourages this development. Up to the present time, theoretical analyses have been restricted to the case of $\alpha$-resorcinol. The next step theoretically would seem to be to confront the theoretical model with the experimental findings and show why the proposed theoretical mechanisms inhibit dislocation formation and propagation. At least we now have a step forward to ask also whether or not the same theoretical methodology is applicable to the present material and of course the numerous others that behave similarly. ${ }^{48-51}$

Finally, it should also be pointed out that there still exist reports of limited growth on the apparently dead $+c$ direction faces that need to be resolved. These include

- The two-dimensional growth of $\alpha$-resorcinol from benzene solutions by Wells in the original paper on this matter $^{12-14}$ and the parallel similar growth of $\mathrm{D}, \mathrm{L}$-alanine from solution in water by $\mathrm{Han}$ et al. $^{55}$

- The growth by $180^{\circ}$ twinning of $\gamma$-glycine $(S),{ }^{56} \alpha$ resorcinol $(\mathrm{S}+\mathrm{V}),{ }^{22,23}$ and MBANP $(\mathrm{S})^{10}$ during growth from solution (S) and PVT (V).

- The recently reported two-dimensional growth of small (1 mm) methyl-4-nitroaniline crystals from the vapor phase by Hesterberg et al. ${ }^{57}$

- Inhibited directional growth of proteins. ${ }^{58}$

All that can be said on these matters at present is that such growth has been ascribed to early growth at high supersaturations, ${ }^{10,55}$ renucleation at the nongrowing surfaces, ${ }^{10,22,56}$ or surface damage, ${ }^{23}$ but this does not rule out other factors. For the present, one-dimensional growth of larger crystals under normal supersaturation conditions, as in the present case, appears to be the rule for unidimensional growth from melt, vapor, and solution in all reported cases.

Influence of Seeding on Crystal Development. It will have been noted that there is a significant distinction in morphology between the seeded crystals pictured in Figure $3 \mathrm{~d}$ and Figure 4 grown at $\sigma=0.92$ and those grown at similar supersaturations by self-nucleation. The morphology of the product crystal replicates the morphology achieved by selfnucleation at much higher supersaturations $(\sigma=0.98)$. The crystal also shows the strain and imperfection exhibited by such crystals. Such differences between the behavior of self-nucleated growth and seeded growth have been found to be common occurrences in the case of growth of crystals from solution. The differences are believed to arise from the fact that nucleation requires the imposition of a much higher supersaturation than the eventual growth process. Thus, following self-nucleation, the initial nucleus will grow very rapidly, decreasing the supersaturation to a lower value than originally anticipated and which then controls subsequent growth. A crystal self-nucleated at a set nucleation supersaturation will exhibit a morphology more characteristic of growth at a lower growth supersaturation than the nucleation supersaturation. A seeded procedure does not suffer from this change, the set supersaturation being that which will drive the subsequent growth process.

The present micromorphological data on seeded growth imply that self-nucleation at $\sigma=0.93$ to 0.96 yields a crystal that grows by a dislocation-nucleated BCF mechanism. The direct study as demonstrated in Figures 5 and 6 however reveals that similar mechanistic processes allow seeded growth to occur at much lower supersaturations $(\sigma=0.32)$. Perhaps the drop is reasonable, but it seems strange, especially since the nucleation supersaturation is apparently held at the higher point during that subsequent post-nucleation growth. This distinction exactly parallels the equivalent situation in solution growth, where growth by self-nucleation regularly yields crystals with wideranging morphologies very different to predicted morphologies. ${ }^{59}$ It thus seemed to be appropriate then to refer to the selfnucleated crystal supersaturations indicated in this article as 
nucleation supersaturations to distinguish them from growth supersaturations. This realization in no way negates the results of experiments on the relative supersaturation dependence of the crystal morphology. It simply draws attention to the fact that the growth supersaturation, while it is probably increasing in each successive experiment, cannot be absolutely defined. This conclusion has ramifications for the study of morphological changes in solution growth, where the self-nucleation technique is a predominant method for examining the influence of ranges of solvents on the morphology of a crystallized product. It would suggest that success of such studies would be best achieved using better controllable seeded growth. ${ }^{50}$

Growing Large Crystals. Despite the apparent setbacks of the present method from the viewpoint of defining the absolute definition of the fundamental supersaturation conditions for the growth of large crystals, PVT growth by self-nucleation as described remains a viable method for the production of highquality crystals in sizes of $1-2 \mathrm{~cm}$ dimensions. The X-ray topographic examinations show that the bipyramidal crystals produced at intermediate supersaturations are of equivalent quality to those produced by solution growth techniques.

What is now required is the definition of the true growth supersaturation to be applied to the seeded growth experiment using the method initially developed by Catano and Kun ${ }^{46}$ that will allow the production of samples of the promised quality of the self-nucleated crystals. A step in this direction for MHB is described in a following paper describing the kinetics of vapor phase growth for this material and which confirms the true growth supersaturation range for perfect growth. ${ }^{47}$

\section{ASSOCIATED CONTENT}

\section{S Supporting Information}

The Supporting Information is available free of charge on the ACS Publications website at DOI: 10.1021/acs.cgd.8b01728.

Temperature gradient tests, the transparent growth system, the seeded growth system (PDF)

\section{AUTHOR INFORMATION}

\section{Corresponding Author}

*Phone: +44 (0)141 9563978. E-mail: j.n.sherwood@strath.ac. uk.

\section{ORCID}

Ranko M. Vrcelj: 0000-0001-6327-2300

John N. Sherwood: 0000-0003-3078-0928

\section{Present Addresses}

${ }^{1}$ Dr. W. Hou, St. Jude Medical Centre, 15900 Valley View Court. Sylmar, CA91342, USA. wenbo11@yahoo.com.

${ }^{2}$ Dr R. I. Ristic, Dept. of Chemical and Process Engineering, University of Sheffield, Sheffield, S1 3JD, UK. r.i.ristic@ sheffield.ac.uk.

${ }^{3}$ Prof K. Srinivasan, Crystal Growth Laboratory, Department of Physics, Barathiar University, Coimbatore-641046, TN, India. nivas55yahoo.com.

${ }^{4}$ Dr $\bar{R}$. M. Vrcelj, Centre for Defense Chemistry, Defense Academy of the UK, Cranfield University, Shrivenham, SN6 8LA, UK. Ranko.Vrcelj@cranfield.ac.uk.

${ }^{5}$ Dr R. B. Hammond, School of Chemical and Process Engineering, University of Leeds, Leeds LS2 9JT, UK. R.B. Hammond@leeds.ac.uk.

\section{Notes}

The authors declare no competing financial interest.

\section{ACKNOWLEDGMENTS}

W.H. wishes to express his grateful thanks to the Univ. of Strathclyde of the award of a CVCP research grant to allow him to perform this research. The EPSRC is thanked for the provision of additional facilities provided by their general financial support of the Strathclyde laboratories, including the use of Station 7.6 of the UK Synchrotron Radiation Source Daresbury, UK, where the synchrotron topographic studies were performed. The authors thank the Director and his Staff for their kind help in this respect.

\section{REFERENCES}

(1) Chemla, D. S., Zyss, J., Eds. Non-Linear Optical Properties of Organic Molecules and Crystals; Academic Press: Orlando, FL, 1987; Vols. I and II.

(2) Kobayashi, T., Ed. Non-linear Optics of Organic and Semiconductors in Physics; Springer Verlag: Berlin, Germany, 1988.

(3) Hann, R. H., Bloor, D., Eds. Organic Materials for Non-linear Optics; The Royal Society of Chemistry: London, UK, 1991.

(4) Zyss, J., Ed. Molecular Non-linear Optics: Materials, Physics and Devices; Academic Press, 1993.

(5) Pugh, D.; Morley, J. O. Molecular Hyperpolarizabilities of Organic Materials; Academic Press, 1987; pp 193-226.

(6) Nicoud, J. F.; Twieg, R. J. Design and Synthesis of Organic Molecular Compounds for Efficient Second Harmonic Generation; Academic Press, 1987; pp 227-296.

(7) Bailey, R. T.; Cruickshank, F. R.; Pugh, D.; Sherwood, J. N. Growth, Perfection and Properties of Organic Non-linear Materials. Acta Crystallogr., Sect. A: Found. Crystallogr. 1991, A47, 145-155.

(8) Bailey, R. T.; Cruickshank, F. R.; Pugh, D.; Sherwood, J. N. Organic Electro-optic and Nonlinear Materials. Int. J. Optoelectronics 1990, 5, 89-102.

(9) Sherwood, J. N.; et al. Molecular Non-linear Optical Materials. Philos. Trans. R. Soc., A 1990, A330, 127-140.

(10) Halfpenny, P. J.; Morrison, H.; Ristic, R. I.; Shepherd, E. A.; Sherwood, J. N.; Simpson, G. S.; Yoon, C. S. The Growth of Large Single Crystals of the Organic Non-linear Optical Material 2-(-methyl benzyl amino)-5-nitropyridine (MBANP). Proc. R. Soc. London, Ser. A 1993, A440, 683-699.

(11) Halfpenny, P. J.; Sherwood, J. N.; Simpson, G. S. The Growth and Perfection of Crystals of Non-linear Optical Materials. In Nonlinear Optical Materials: Principals and Applications; Degiorgio, V.; Flytzanis, C., Eds.; IOS Press: Amsterdam, The Netherlands, 1995; pp 131-174.

(12) Wells, A. F. Crystal Habit and Internal Structure - I. Philos. Mag. 1946, 37, 184-199.

(13) Wells, A. F. Crystal Habit and Internal Structure - II. Philos. Mag. 1946, 37, 217-236.

(14) Wells, A. F. Abnormal and Modified Crystal Growth. Discuss. Faraday Soc. 1949, 5, 197-201.

(15) Davey, R. J.; Milisavljevic, B.; Bourne, J. R. Solvent Interactionsat Crystal Surfaces - The kinetic story of alpha-resorcinol. J. Phys. Chem. 1988, 92, 2032-2036.

(16) Davey, R. J. The Role of Solvent in Crystal Growth from Solution. J. Cryst. Growth 1986, 76 (3), 637-644.

(17) ter Horst, J. H.; Geertman, R. M.; van Rosmalen, G. M. The Effect of Solvent on Crystal Morphology. J. Cryst. Growth 2001, 230, 277-284.

(18) Lahav, M.; Leiserowitz, L. The Effect of Solvent on Crystal Growth and Morphology. Chem. Eng. Sci. 2001, 56, 2245-2253.

(19) Wireko, F. C.; Shimon, L. J. W.; Frolow, F.; Berkovitch Yellin, Z.; Lahav, M.; Leiserowitz, L. Effect of Solvent on the Growth of OrganicCrystals 1. The riddle of alpha-resorcinol. J. Phys. Chem. 1987, 91, 472-481.

(20) Wang, J. L.; Leiserowitz, L.; Lahav, M. A Correlation betweenSurface Wettability and Solvent Effect on Crystal Growth - 
The Normal-octyl-D-gluconamide methanol system. J. Phys. Chem. 1992, 96, 15-16.

(21) Liu, X. Y.; Boek, E. S.; Briels, W. J.; Bennema, P. Prediction ofCrystal Growth Morphology Based on Structural Analysis of the Solid FluidInterface. Nature 1995, 374, 342-345.

(22) Srinivasan, K.; Sherwood, J. N. Asymmetric Growth of alphaResorcinol Crystals: Comparison of growth from the vapor phase and from aqueous solution. Cryst. Growth Des. 2005, 5, 1359-1370.

(23) Srinivasan, K.; Sherwood, J. N. Asymmetric Growth of alphaResorcinol Crystals: In situ studies of crystal growth from the vapor phase. Cryst. Growth Des. 2011, 11, 5010-5018.

(24) Hussain, M.; Anwar, J. The Riddle of Resorcinol Crystal Growth Revisited: Molecular dynamics simulations of alpha-resorcinol crystal water interface. J. Am. Chem. Soc. 1999, 121, 8583-8591.

(25) Weissbuch, I.; Leiserowitz, L.; Lahav, M. Self-poisoning at $\{011\}$ Faces of alpha-Resorcinol Crystals may explain its Unidirectional Growth in the Vapour Phase: A molecular modelling study. Cryst. Growth Des. 2006, 6, 625-628.

(26) Anwar, J.; Chatchawalsaisin, J.; Kendrick, J. Asymmetric Crystal Growth of alpha-Resorcinol from the Vapor Phase: Surface reconstruction and conformational change are the culprits. Angew. Chem., Int. Ed. 2007, 46, 5537-5540.

(27) Ectors, P.; Sae-Tang, W.; Chatchawalsaisin, J.; Zahn, D.; Anwar, J. The Molecular Mechanism of $\alpha$-Resorcinol's Asymmetric Crystal Growth from theMelt Jul. Cryst. Growth Des. 2015, 15, 4026-4031.

(28) Jerphagnon, J. Optical Second Harmonic Generation in Isocyclic and Heterocyclic Compounds. IEEE J. Quantum Electron. 1971, 7, 4243.

(29) Sreeramana Aithal, P.; Mohan Rao, P. Novel Non-linear Optical Crystal of Methyl-p-hydroxybenzoate. J. Cryst. Growth 1995, 153, 6062.

(30) Li, Z.; Wu, B.; Su, G. Non-linear Optical, Optical, and Crystallographic Properties of Methyl-p-hydroxybenzoate. J. Cryst. Growth 1997, 178, 539-544.

(31) Lakshmana Perumal, C. K.; Arulchakkaravarthi, A.; Rajesh, N. P.; Santhanaraghavan, P.; Ramasamy, P. Microhardness and Slip Systems of Solution Grown MHB Crystals. Mater. Lett. 2002, 56, 578-586.

(32) Sritharan, K.; Manikandan, V.; Srinivasan, K. A Newer Approach to Growing Methyl-p-hydroxy benzoate pMHB Single Crystals Without a Polymorphic Change in their Form. CrystEngComm 2016, $18,8237-8245$.

(33) Soni, M. G.; Taylor, S. L.; Greenberg, N. A.; Burdock, G. A. Evaluation of the Health Aspects of Methyl Paraben: a review of the published literature. Food Chem. Toxicol. 2002, 40, 1335-1373.

(34) McArdle, B. J.; Sherwood, J. N. In Advanced Crystal Growth; Dryburgh, P.; Cockayne, B.; Barraclough, K. G., Eds.; Prentice-Hall, New York, 1987.

(35) Lin, X. T. J. Struct. Chem. 1983, 3, 213 (in Chinese). .

(36) Vujovic, D.; Nassimbeni, L. R. Methyl Paraben: A New Polymorph. Cryst. Growth Des. 2006, 6, 1595-1597.

(37) Nath, N. K.; Aggarwal, H.; Nangia, A. Crystal Structure of Methyl Paraben Polymorph II. Cryst. Growth Des. 2011, 11, 967-971.

(38) Gelbrich, T.; Braun, D. E.; Ellern, A.; Griesser, U. J. Four Polymorphs of Methyl Paraben: Structural Relationships and Relative Energy Differences. Cryst. Growth Des. 2013, 13, 1206-1217.

(39) Perlovich, G. L.; Rodionov, S. L.; Bauer-Brandl, A. Thermodynamics of Solubility, Sublimation and Solvation Processes of Parabens. Eur. J. Pharm. Sci. 2005, 24, 25-33.

(40) Sherwood, J. N.; Horton, P. N.; Coles, S. J.; Vrcelj, R. M. The Determination of the Absolute Configuration of Some Non-Linear Optical Materials and the Relationship to Anisotropic Crystal Growth. In preparation.

(41) Tanner, B. K. X-ray Diffraction Topography; Pergamon Press: Oxford, U.K., 1976.

(42) Lang, A. R. Defect Visualisation-Individual Defects. In Characterisation of Crystal Growth Defects by X-ray Methods-NATO Science Series B, Tanner, B. K.; Bowen, D. K., Eds.; Springer, 1980; Vol 63.
(43) Clydesdale, G.; Doherty, R.; Roberts, K. J. HABIT95, Quantum Chemistry Program Exchange. Program No. 670; QC Plumbing \& Electrical: Bloomington, IN, 1996, 61, 1-11.

(44) Mayo, S. L.; Olafson, B. D.; Goddard, W. A. DREIDING: A Generic Force Field for Molecular Simulations. J. Phys. Chem. 1990, 94, 8897-8909.

(45) Piper, W. W.; Polich, S. J. Vapour Phase Growth of II-VI Compounds. J. Appl. Phys. 1961, 32, 1278-1279.

(46) Catano, A.; Kun, Z. K. Growth and Characterisation of ZnSe and Homogeneous ZnSxSe-x Crystals. J. Cryst. Growth 1976, 33, 324-330.

(47) Hou, W.; Ristic, R. I.; Sherwood, J. N. Crystal Growth of the Acentric Organic Non-linear Optical Material Methyl-p-hydroxybenzoate (MHB): Kinetics of Crystal Growth. To be published.

(48) Raghavan, S. L.; Ristic, R. I.; Sheen, D. B.; Sherwood, J. N.; Trowbridge, L.; York, P. The Morphology of Crystals of R-Lactose Hydrate Grown from Aqueous Solution. J. Phys. Chem. B 2000, 104, 12256-12262.

(49) Shepherd, E. E. A.; Sherwood, J. N.; Simpson, G. S.; Yoon, C. S. The Growth and Perfection of Organic Non-linear Optical Materials 4nitro-4'-methylbenzylidine aniline (NMBA). J. Cryst. Growth 1991, 113, 360-370.

(50) Shekunov, B. Y.; Shepherd, E. E. A.; Sherwood, J. N.; Simpson, G. S. Growth and Perfection of Organic Non-linear Optical Materials: Kinetics and Mechanism of the Growth of N-(4-Nitrophenyl-prolinol (NPP) Crystals from Ethanol and Toluene Solution. J. Phys. Chem. 1995, 99, 7130-7136.

(51) Hou, W.; Ristic, R. I.; Srinivasan, K.; Sheen, D. B.; Sherwood, J. N. Crystal Growth of The Acentric Organic Non-Linear Optical Material Methyl-p-Hydroxybenzoate (MHB): Comparison of growth from solution and the vapour phase: In preparation.

(52) Chernov, A. A. Formation of Crystals in Solution. Contemp. Phys. 1989, 30, 251-276.

(53) van Enckevort, W. J. P. Growth Steps on Crystal Surfaces: Their Observation and their Interpretations. Current Topics in Crystal Growth Research 1995, 2, 535-551.

(54) Lovette, M. A.; Browning, A. R.; Griffin, D. W.; Sizemore, J. P.; Snyder, R. C.; Doherty, M. F. Crystal Shape Engineering. Ind. Eng. Chem. Res. 2008, 47, 9812-33.

(55) Han, G.; Chow, P. S.; Tan, R. B. H. Precise Habit Modification of Polar dl-Alanine Crystals by Control of Supersaturation. Cryst. Growth Des. 2011, 11, 3941-3946.

(56) Srinivasan, K.; Renuka Devi, K.; Anbuchudar Azhagan, S. Characterisation of $\alpha$ and $\gamma$ Polymorphs of Glycine Crystallsised from Water/Ammonia solutions. Cryst. Res. Technol. 2011, 46, 159-165.

(57) Hesterberg, R.; Bonin, M.; Sommer, M.; Burgener, M.; Trusch, B.; Damjanovic, D.; Hulliger, J. Vapour Growth, Morphology, Absolute Structure and Pyroelectric Coefficient of meta-Nitroaniline Single Crystals. J. Appl. Crystallogr. 2019, 52, 564-570.

(58) Heijna, M. C. R.; Van Enckevort, W. J. P.; Vlieg, E. Inhibited directional growth of lysozyme crystals. Cryst. Growth Des. 2008, 8, 270-274.

(59) Gallagher, H. G.; Sherwood, J. N.; Vrcelj, R. M. The Growth and Perfection of $\beta$-Cyclotetramethylene-tetranitramine (HMX) Studied by Laboratory and Synchrotron X-Ray Topography. J. Cryst. Growth 2017, 475, 192-201. 